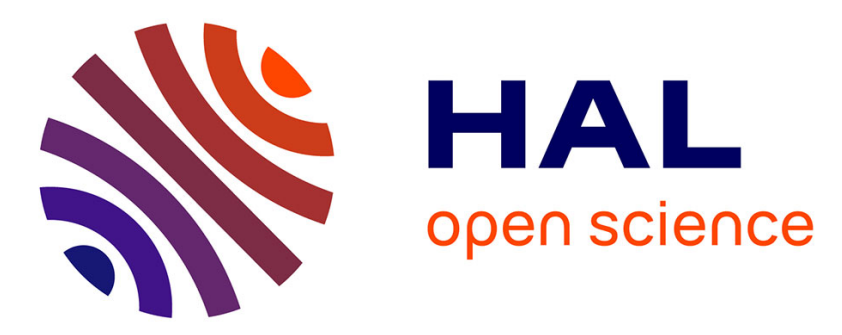

\title{
Kolmogorov and Kelvin wave cascades in a generalized model for quantum turbulence
}

Nicolás P Müller, Giorgio Krstulovic

\section{To cite this version:}

Nicolás P Müller, Giorgio Krstulovic. Kolmogorov and Kelvin wave cascades in a generalized model for quantum turbulence. Physical Review B, 2020, 102, 10.1103/physrevb.102.134513 . hal-03054358

\section{HAL Id: hal-03054358 \\ https://hal.science/hal-03054358}

Submitted on 11 Dec 2020

HAL is a multi-disciplinary open access archive for the deposit and dissemination of scientific research documents, whether they are published or not. The documents may come from teaching and research institutions in France or abroad, or from public or private research centers.
L'archive ouverte pluridisciplinaire HAL, est destinée au dépôt et à la diffusion de documents scientifiques de niveau recherche, publiés ou non, émanant des établissements d'enseignement et de recherche français ou étrangers, des laboratoires publics ou privés. 


\title{
Kolmogorov and Kelvin wave cascades in a generalized model for quantum turbulence
}

\author{
Nicolás P. Müller $\odot$ and Giorgio Krstulovic $\odot$ \\ Université Côte d'Azur, Observatoire de la Côte d'Azur, CNRS, Laboratoire Lagrange, \\ Boulevard de l'Observatoire CS 34229 - F 06304 NICE Cedex 4, France
}

(Received 1 July 2020; revised 16 September 2020; accepted 11 October 2020; published 28 October 2020)

\begin{abstract}
We performed numerical simulations of decaying quantum turbulence by using a generalized Gross-Pitaevskii equation that includes a beyond mean field correction and a nonlocal interaction potential. The nonlocal potential is chosen in order to mimic He II by introducing a roton minimum in the excitation spectrum. We observe that at large scales the statistical behavior of the flow is independent of the interaction potential, but at scales smaller than the intervortex distance a Kelvin wave cascade is enhanced in the generalized model. In this range, the incompressible kinetic energy spectrum obeys the weak wave turbulence prediction for Kelvin wave cascade not only for the scaling with wave numbers but also for the energy flux and the intervortex distance.
\end{abstract}

DOI: 10.1103/PhysRevB.102.134513

\section{INTRODUCTION}

One of the most fundamental phase transitions in low temperature physics is the Bose-Einstein condensation [1]. It occurs when a fluid composed of bosons is cooled down below a critical temperature. In that state, the system has long-range order and can be described by a macroscopic wave function. One of the most remarkable properties of a BoseEinstein condensate (BEC) is that it flows with no viscosity. Well before the first experimental realization of a BEC by Anderson et al. [2], Kaptiza and Allen discovered that helium becomes superfluid below $2.17 \mathrm{~K}[3,4]$. A couple of years later, London suggested that superfluidity is intimately linked to the phenomenon of Bose-Einstein condensation [5]. Since then, superfluid helium and BECs made of atomic gases have been extensively studied, both theoretical and experimentally. In particular, the fluid dynamics aspect of quantum fluids has renewed interest due the impressive experimental progress of the last fifteen years. Today it is possible to visualize and follow the dynamics of quantum vortices, one the most fundamental excitations of a quantum fluid $[6,7]$.

Quantum vortices are topological defects of the macroscopic wave function describing the superfluid. They are nodal lines of the wave function and they manifest points and filaments in two and three dimensions, respectively. To ensure the monodromy of the wave function, vortices have the topological constraint that the circulation (contour integral) of the flow around the vortex must be a multiple of the Feynman-Onsager quantum of circulation $\kappa=h / m$, where $h$ is the Planck constant and $m$ is the mass of the Bosons constituting the fluid [1]. In superfluid helium their core size is of the order of $1 \AA$ whereas in atomic BECs is typically of the order of microns [8]. Quantum vortices interact with other vortices similarly to classical ones. They move thanks to their self-induced velocity and interact with each other by hydrodynamics laws [9]. Unlike ideal classical vortices described by Euler equations, quantum vortices can reconnect and change their topology despite the lack of viscosity of the fluid [10].

At scales much larger than the mean intervortex distance $\ell$, the quantum nature of vortices is not very important as many individual vortices contribute to the flow. One could expect then that the flow is similar, in some sense, to a classical one. Indeed, if energy is injected at large scales, a classical Kolmogorov turbulent regime emerges. Such a regime has been observed numerically [11-13] and experimentally in superfluid helium $[14,15]$. In a three-dimensional turbulent flow, energy is transferred towards small scales in a cascade process [16]. In a low temperature turbulent superfluid, when energy reaches the intervortex distance, energy keeps being transferred to even smaller scales where it can be efficiently dissipated by sound emission. The mechanisms responsible for this transfer are vortex reconnections and the wave turbulence cascade of Kelvin waves, that have its origin in the quantum nature of vortices [17].

Describing a turbulent superfluid is not an easy task, in particular for superfluid helium. One of the main reasons is the gigantic scale separation existing between the vortex core size and the typical size of experiments, currently of the order of centimeters or even meters [18]. Their theoretical description began at the beginning of the 20th century by the pioneering works of Landau and Tisza where superfluid helium was modeled by two immiscible fluid components [19]. In this two-fluid model, the thermal excitations constitute the so-called normal fluid that is described by the Navier-Stokes equations whereas the superfluid component is treated as an inviscid fluid. It was later realized that the thermal excitations interact with superfluid vortices through scattering processes that lead to a coupling of both components by mutual friction forces [19]. Today the two-fluid description, known as the Hall-Vinen-Bekarevich-Khalatnikov model is understood as a coarse-grained model where scales smaller than the intervortex distance are not considered. The quantum nature aspects 
of superfluid vortices are therefore lost. However, this model remains useful for describing the large scale dynamics of finite temperature superfluid helium. An alternative model was introduced by Schwarz [9], where vortices are described by vortex filaments interacting through regularized Biot-Savart integrals. However, the reconnection process between lines needs to be modeled in an ad-hoc manner and by construction the model excludes the dynamics of a superfluid at scales smaller than the vortex core size. Finally, for weakly interacting BECs in the limit of low temperature, a model of different nature can be formally derived which is the Gross-Pitaevskii (GP) equation, obtained from a mean field theory [1]. This model naturally contains vortex reconnections $[10,20]$, sound emission [21,22], and is known to also exhibit a Kolmogorov turbulent regime at scales much larger than the intervortex distance [11]. Although this model is expected to provide some qualitative description of superfluid helium at low temperatures, it lacks of several physical ingredients. For instance, in GP, density excitations do not present any roton minimum as it does superfluid helium, where interactions between boson are known to be much stronger than in GP [23]. However, there have been some successful attempts to include such effects in the GP model. For instance, a roton minimum can be easily introduced in GP by using a nonlocal potential that models a long-range interaction between bosons [24-26]. The stronger interaction of helium can also be included phenomenologically by introducing high-order terms in the GP Hamiltonian. Note that these terms can be derived as beyond mean field corrections [27]. Some generalized version of the GP model has been used to study the vortex solutions $[28,29]$ and some dynamical aspects such as vortex reconnections [26]. Intuitively, for a turbulent superfluid, we can expect that such generalization of the GP model might be important at scales smaller than the intervortex distance and with less influence at scales at which Kolmogorov turbulence is observed.

In this work, we study quantum turbulent flows by performing numerical simulations of a generalized GrossPitaevskii (gGP) equation. We compare the effect of highorder nonlinear terms and the effect of a nonlocal interaction potential in the development and decay of turbulence at scales both larger and smaller than the intervortex distance. Remarkably, by modeling superfluid helium with a nonlocal interaction potential and including high-order terms, the range where a Kelvin wave cascade is observed is extended and becomes manifest. Using the dissipation (or rate of transfer) of incompressible kinetic energy we are able to show that the weak wave turbulence results [30] are valid not only to predict the scaling with wave number but also with the energy flux and the intervortex distance.

The paper is organized as follows. Section II introduces the gGP model and discusses its basic properties and solutions. It also discusses how the vortex profile is modified in this generalized model. All useful definitions to study turbulence are also given here. Section III gives a brief overview of the predictions of quantum turbulence and the numerical methods used in this work. Also, it includes the results of different simulations at moderate and high resolutions by varying the different parameters of the beyond mean field correction and the introduction of a nonlocal potential. Finally in Sec. IV we present our conclusions.

\section{THEORETICAL DESCRIPTION OF SUPERFLUID TURBULENCE}

In this section we introduce the generalized GrossPitaevskii model used in this work. We also discuss and review some of the basic properties of the model such as its elementary excitations and its hydrodynamic description.

\section{A. Model}

The Gross-Pitaevskii equation describes the low temperature dynamics of weakly interacting bosons of mass $m$

$$
i \hbar \frac{\partial \psi}{\partial t}=-\frac{\hbar^{2}}{2 m} \nabla^{2} \psi-\mu \psi+g|\psi|^{2} \psi
$$

where $\psi$ is the condensate wave function, $\mu$ the chemical potential, $\hbar=h /(2 \pi)$, and $g=4 \pi \hbar^{2} a_{s} / m$ is the coupling constant fixed by the $s$-wave scattering length $a_{s}$ that models a local interaction between bosons. Note that the use of a local potential assumes a weak interaction between bosons, which certainly is not the case for other systems like He II and for dipolar gases [31].

A generalized model that is able to describe more complex systems can be obtained by considering a nonlocal interaction between bosons. With proper modeling [24-26], density excitations exhibit a roton minimum in their spectrum as the one observed in He II [23]. It also describes well the behavior of dipolar condensates [32,33]. In helium and other superfluids, the interaction between bosons is stronger and high-order nonlinearities are needed for proper modeling. For instance, in helium high-order terms are considered to mimic its equation of state [25] and in dipolar BECs beyond mean field terms are needed to describe the physics of recent supersolid experiments [34].

We consider the generalized Gross-Pitaevskii (gGP) model written as

$$
\begin{aligned}
i \hbar \frac{\partial \psi}{\partial t}= & -\frac{\hbar^{2}}{2 m} \nabla^{2} \psi-\mu(1+\chi) \psi \\
& +g\left(\int V_{\mathrm{I}}(\boldsymbol{x}-\boldsymbol{y})|\psi(\boldsymbol{y})|^{2} d^{3} y\right) \psi+g \chi \frac{|\psi|^{2(1+\gamma)}}{n_{0}^{\gamma}} \psi
\end{aligned}
$$

where $\gamma$ and $\chi$ are two dimensionless parameters that determine the order and amplitude of the high-order terms, respectively. The interaction potential $V_{\mathrm{I}}$ is normalized such that $\int V_{\mathrm{I}}(\boldsymbol{x}) d^{3} x=1$. The chemical potential and the interaction coefficient of the high-order term have been renormalized such that $\left|\psi_{0}\right|^{2}=n_{0}=\mu / g$ is the density of particles for the ground state of the system for all values of parameters. The GP equation (1) is recovered by simply setting $V_{\mathrm{I}}(\boldsymbol{x}-\boldsymbol{y})=\delta(\boldsymbol{x}-\boldsymbol{y})$ and $\chi=0$. The gGP equation is not intended to be a first principle model of superfluid helium, but it has the advantage of at least introducing in a phenomenological manner some important physical aspects of helium. 


\section{B. Density waves}

The dispersion relation of the GP model is easily obtained by linearizing equation (1) about the ground state. The waves obey the Bogoliubov dispersion that reads

$$
\omega_{B}(k)=c_{0} k \sqrt{\frac{\xi_{0}^{2} k^{2}}{2}+1}
$$

where $k$ is the wave number, $c_{0}=\sqrt{g n_{0} / m}$ is the speed of sound of the superfluid, and $\xi_{0}=\hbar / \sqrt{2 m g n_{0}}$ is the healing length at which dispersive effects become important. The healing length also fixes the vortex core size.

A similar calculation leads to the Bogoliubov dispersion relation in the case of the gGP model (2)

$$
\omega(k)=c k \sqrt{\frac{\xi^{2} k^{2}}{2}+\frac{\hat{V}_{\mathrm{I}}(k)+\chi(\gamma+1)}{1+\chi(\gamma+1)}}
$$

where $\hat{V}_{\mathrm{I}}(\boldsymbol{k})=\int e^{i \boldsymbol{k} \cdot \boldsymbol{r}} V_{\mathrm{I}}(\boldsymbol{r}) d^{3} r$ is the Fourier transform of the interaction potential normalized such that $\hat{V}_{\mathrm{I}}(k=0)=1$. The inclusion of beyond mean field terms and a nonlocal potential yields to a renormalized speed of sound and healing length. They are given in terms of $c_{0}$ and $\xi_{0}$ by

$$
\begin{gathered}
c=c_{0} \sqrt{1+\chi(\gamma+1)} \\
\xi=\frac{\xi_{0}}{\sqrt{1+\chi(\gamma+1)}} .
\end{gathered}
$$

Note that, in what concerns low amplitude density waves, the effect of high-order terms is a simple renormalization of the healing length and the speed of sound. Depending on the shape and properties of the nonlocal potential, the dynamics and steady solutions can be drastically modified. Note that the product between $c$ and $\xi$ remains constant because it is related to the quantum of circulation $\kappa=h / m=c \xi 2 \pi \sqrt{2}=$ $c_{0} \xi_{0} 2 \pi \sqrt{2}$.

In order to be able to compare systems with different types of interactions, it is convenient to rewrite Eq. (2) in terms of its intrinsic length $\xi$ and speed of sound $c$ and the bulk density $n_{0}$. The gGP model then becomes

$$
\partial_{t} \psi=-i \frac{c}{\xi \sqrt{2}(1+\chi(\gamma+1))}\left[-(1+\chi(\gamma+1)) \xi^{2} \nabla^{2} \psi-(1+\chi) \psi+\chi \frac{|\psi|^{2(1+\gamma)}}{n_{0}^{1+\gamma}} \psi+\frac{\psi}{n_{0}} \int V_{\mathrm{I}}(\boldsymbol{x}-\boldsymbol{y})|\psi(\boldsymbol{y})|^{2} d^{3} y\right]
$$

The only dimensional parameters of the model are the speed of sound $c$, the healing length $\xi$, and the bulk density $n_{0}$. They can be absorbed by a trivial rescaling of time, length, and density. The dimensionless parameters $\chi, \gamma$ and the nonlocal potential $V_{\mathrm{I}}$ should be chosen to model the physical system under study. In numerical simulations we will express lengths in units of the healing length $\xi$. A natural time scale to study excitations is the fast turnover time $\tau=\xi / c$. However, this small-scale based time is not appropriate for turbulent flows. For such flows, it is customary to use the large-eddy turnover time corresponding to the typical time of the largest coherent vortex structures. It will be defined later.

\section{Modeling superfluid helium excitations}

In this work, we aim at mimicking some properties of superfluid helium II, in particular, the roton minimum in the dispersion relation. For the sake of simplicity, we use an isotropic nonlocal interaction potential used in previous works [26,28]. With our normalization it reads

$$
\hat{V}_{\mathrm{I}}(\boldsymbol{k})=\left[1-V_{1}\left(\frac{k}{k_{\mathrm{rot}}}\right)^{2}+V_{2}\left(\frac{k}{k_{\mathrm{rot}}}\right)^{4}\right] \exp \left(-\frac{k^{2}}{2 k_{\text {rot }}^{2}}\right),
$$

where $k_{\text {rot }}$ is the wave number associated with the roton minimum and $V_{1} \geqslant 0$ and $V_{2} \geqslant 0$ are dimensionless parameters to be adjusted to mimic experimental dispersion relation of helium II [23]. The effects of different functional forms of the nonlocal potential have been studied in previous works, showing that only a phase shift of $\psi$ and the overall amplitude of the density depend on the precise form of the interaction [29].

In order to compare the dispersion relation (4) with the experimental data [23], we plot the helium dispersion relation in units of the helium healing length $\xi_{\mathrm{He}}=0.8 \AA$ and its turnover time $\tau_{\mathrm{He}}=\xi_{\mathrm{He}} / c_{\mathrm{He}}=3.36 \times 10^{-13} \mathrm{~s}$, where $c_{\mathrm{He}}=238 \mathrm{~m} / \mathrm{s}$ is the speed of sound in He II. The experimental helium dispersion relation is displayed in Fig. 1 as green dotted lines. Note that by definition, the speed of sound of the gGP model and the one helium are equal to 1 (in units of $\xi / \tau$.)

It was reported in Reneuve et al. [26] that introducing a roton minimum in the GP dispersion relation (without beyond mean field terms) that matches helium measurements leads to an unphysical crystallization under dynamical evolution of
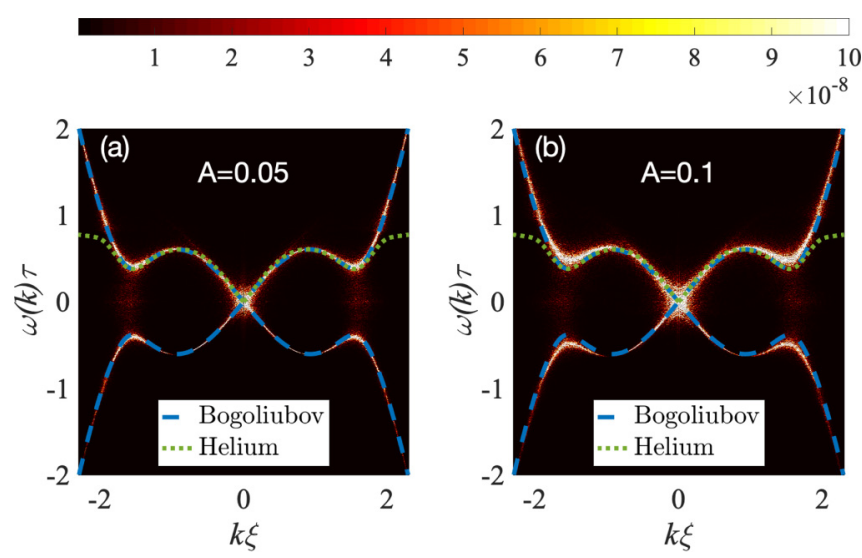

FIG. 1. Spatiotemporal dispersion relation for simulations with $1024^{2}$ grid points with a nonlocal potential and beyond mean field corrections. Light zones correspond to excited frequencies. Figures (a) and (b) correspond to different amplitude of the perturbation $A$, both exhibiting a roton minimum. Experimental observations (green dotted line, see Ref. [23]) and theoretical dispersion following equation (4) (blue dashed line) are shown. 
a vortex. We confirm such behavior in our simulations. In order to avoid such spurious effect of the model, in Ref. [26] the frequency associated to the roton minimum was set to higher values to be able to study vortex reconnections. We have numerically observed that this crystallization takes place even when a first order correction of the beyond mean field expansion is included, with values of $\chi=0.1$ and $\gamma=1$. For this reason, we chose a higher order expansion with $\gamma=2.8$ for the simulations with a nonlocal potential, a value that was already used in the literature to study the vortex density profile in superfluid helium [25]. Furthermore, with this value no crystallization is observed for all test cases and all the simulations performed in this work.

The dispersion relation of a nonlinear wave system can be measured numerically by computing the spatiotemporal spectrum of the wave field [13]. As an example, in Fig. 1 we also display the spatiotemporal spectrum of small density perturbations of a numerical simulation of the gGP model in two dimensions using $1024^{2}$ collocation points and with parameters set to $\gamma=2.8, \chi=0.1, V_{1}=4.54, V_{2}=0.01$, and $k_{\text {rot }} \xi=1.638$ (see details on numerics later in Sec. III), for two different amplitude values $A$. Dark zones indicate that no frequencies are excited, while light zones correspond to the excited ones with the total sum normalized to one. The parameters have been set in a way such that they match qualitatively the dispersion relation measured in helium up to the roton minimum. As expected for weak amplitude waves, the numerical and theoretical dispersion relations coincide. For larger wave amplitudes, theoretical prediction (4) and numerical measurements slightly differ together with an apparent broadening of the curve. This is a typical behavior of nonlinear wave systems [35]. In the following sections, all simulations with a nonlocal interaction are performed with the aforementioned set of parameters.

\section{Hydrodynamic description}

The GP equation maps into a hydrodynamic description by introducing the Madelung transformation

$$
\psi=\sqrt{\rho / m} \exp \left(\frac{i \phi}{\sqrt{2} c \xi}\right)
$$

which allows the mapping of the wave function with the fluid mass density $\rho=m|\psi|^{2}$ and with the fluid velocity $\boldsymbol{v}=\nabla \phi$. Replacing equation (9) into the gGP model (7) two hydrodynamic equations are obtained

$$
\begin{gathered}
\frac{\partial \rho}{\partial t}+\nabla \cdot(\rho \boldsymbol{v})=0 \\
\frac{\partial \phi}{\partial t}+\frac{1}{2}(\nabla \phi)^{2}=-h[\rho]+(c \xi)^{2} \frac{\nabla^{2} \sqrt{\rho}}{\sqrt{\rho}}
\end{gathered}
$$

with

$$
h[\rho]=-c_{0}^{2}(1+\chi)+c_{0}^{2} \frac{V_{\mathrm{I}} * \rho}{\rho_{0}}+c_{0}^{2} \chi\left(\frac{\rho}{\rho_{0}}\right)^{\gamma+1} .
$$

Here $*$ denotes the convolution product and $\rho_{0}=m\left|\psi_{0}\right|^{2}$ is the fluid mass density of the ground state. These equations correspond to the continuity and Bernoulli equations, respectively, of a fluid with an enthalpy per unit of mass $h[\rho][11]$.
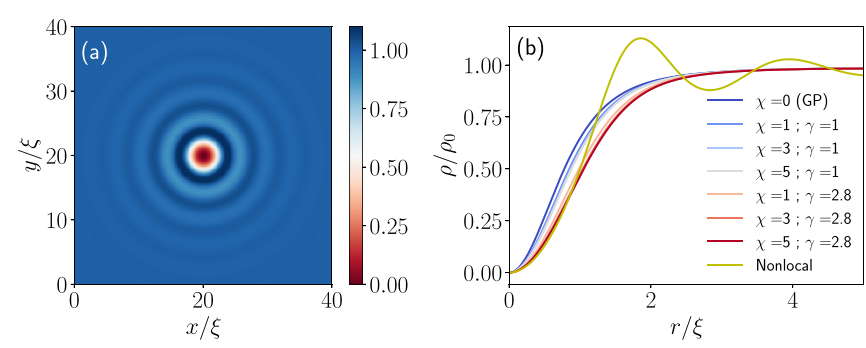

FIG. 2. (a) Mass density of a two-dimensional vortex with a nonlocal potential. (b) Density profile of a vortex for the gGP model with different values of the nonlinearity and a local potential, and a single profile with a nonlocal potential (yellow line). The vortex core size tends to increase with the nonlinearity.

The last term of equation (11) is called the quantum pressure. Note that hydrodynamic pressure is given by

$$
p[\rho]=\frac{c_{0}^{2} \rho}{\rho_{0}}\left[\frac{1}{2} V_{\mathrm{I}} * \rho+\chi \frac{\gamma+1}{\gamma+2} \frac{\rho^{\gamma+1}}{\rho_{0}^{\gamma}}\right] .
$$

As expected, for large amplitude waves, the speed of sound reads $\left.\frac{\partial p}{\partial \rho}\right|_{\rho_{0}}=c_{0}^{2}(1+\chi(\gamma+1))=c^{2}$.

Although the fluid is potential, it admits vortices as topological defects of the wave function. A stationary vortex solution of (7) is a zero of the wave function where the circulation around it is quantized with values $\pm s \kappa$ with $s$ an integer. Because of this last condition, topological defects are also called quantum vortices.

A quantum vortex has a vortex core size of the order of a healing length $\xi$ and depends on the parameters of the gGP model. By replacing the Madelung transformation (9) into the gGP equation (7) and using cylindrical coordinates, a differential equation for the vortex profile is directly obtained

$$
\begin{aligned}
& \frac{1}{r} \frac{d}{d r}\left(r \frac{d R}{d r}\right)+\left\{1-\frac{s^{2} \xi_{0}^{2}}{r^{2}}-V_{\mathrm{I}} * R^{2}+\chi\left(1-R^{2 \gamma+2}\right)\right\} \frac{R}{\xi_{0}^{2}} \\
& \quad=0,
\end{aligned}
$$

where $R(r)=\sqrt{\rho(r) / \rho_{0}}$ defines the density profile of the vortex line in the radial direction $r$.

Figure 2(a) displays the mass density of a two-dimensional vortex in the case where the nonlocal interaction potential is included. The roton minimum introduces some density fluctuations around the center of the vortex which is a well-known pattern. The effect of a nonlocal potential has already been studied before, for example its interaction with an obstacle [24], the dynamics of vortex rings [25], and in reconnection processes [26]. Figure 2(b) shows the radial dependence of the density profile of a vortex for different parameters of the gGP model. Numerical simulations were performed with $4096^{2}$ grid points with standard numerical methods (see Sec. III B for details). Even though all curves tend to collapse when plotted as a function of the healing length $\xi$, the vortex core size slightly increases (in units of $\xi$ ) when the nonlinearity of the system is increased. Note that for the present range of parameters, $\xi_{0} / \xi$ varies in the range $(1,4.4)$. The relatively good collapse of the vortex core size thus justifies the choice of $\xi$ to parametrize the gGP model while varying the beyond mean field parameters. 


\section{Energy decomposition and helicity in superfluids}

It is convenient to write the free energy per unit of mass $\mathcal{F}$ of a quantum fluid such that it vanishes when evaluated in the ground state of the system $\left(\psi=\sqrt{\rho_{0} / m}=\sqrt{n_{0}}\right)$. For the gGP model in equation (7), it is given by

$$
\begin{aligned}
\mathcal{F}= & \frac{c_{0}^{2}}{n_{0} V} \int\left[\xi_{0}^{2}|\nabla \psi|^{2}+\frac{|\psi|^{2}}{2 n_{0}}\left(V_{\mathrm{I}} *|\psi|^{2}\right)-(1+\chi)|\psi|^{2}\right. \\
& \left.+\frac{\chi|\psi|^{2(\gamma+2)}}{n_{0}^{\gamma+1}(\gamma+2)}+\frac{n_{0}}{2}+\chi n_{0} \frac{\gamma+1}{\gamma+2}\right] d^{3} r
\end{aligned}
$$

with $V$ the volume of the fluid. Following standard procedures applied in simulations of GP quantum turbulence [11], the free energy can be decomposed as $\mathcal{F}=$ $E_{\text {kin }}^{I}+E_{\text {kin }}^{C}+E_{\mathrm{q}}+E_{\text {int }}$ where $E_{\text {kin }}^{\mathrm{I}}=\frac{1}{V \rho_{0}} \int\left([\sqrt{\rho} \boldsymbol{v}]^{\mathrm{I}}\right)^{2} d^{3} r$, $E_{\text {kin }}^{\mathrm{C}}=\frac{1}{V \rho_{0}} \int\left([\sqrt{\rho} \boldsymbol{v}]^{\mathrm{C}}\right)^{2} d^{3} r$, and $E_{\mathrm{q}}=\frac{c^{2} \xi^{2}}{V \rho_{0}} \int|\nabla \sqrt{\rho}|^{2} d^{3} r$, with $[\sqrt{\rho} \boldsymbol{v}]^{\mathrm{I}}$ the regularized incompressible velocity obtained via the Helmholtz decomposition and $[\sqrt{\rho} \boldsymbol{v}]^{\mathrm{C}}=$ $\sqrt{\rho} \boldsymbol{v}-[\sqrt{\rho} \boldsymbol{v}]^{\mathrm{I}}$ the compressible one. The internal energy per unit of volume is defined in the gGP model as

$$
\begin{aligned}
E_{\text {int }}= & \frac{c_{0}^{2}}{V \rho_{0}} \int\left[\frac{1}{2 \rho_{0}}\left(\rho-\rho_{0}\right) V_{\mathrm{I}} *\left(\rho-\rho_{0}\right)\right. \\
& \left.+\left(\frac{\rho}{\rho_{0}}\right)^{\gamma+1} \frac{\chi \rho}{\gamma+2}-\chi \rho+\chi \frac{\gamma+1}{\gamma+2} \rho_{0}\right] d^{3} r .
\end{aligned}
$$

Note that $E_{\text {int }}=0$ if $\rho=\rho_{0}$. The corresponding energy spectra are defined in a straightforward way for the quadratic quantities [11]. For the internal energy spectrum, it is defined as follows

$$
\begin{aligned}
E_{\text {int }}(k)= & \frac{c_{0}^{2}}{V \rho_{0}} \int\left[\frac{1}{2 \rho_{0}}\left(\widehat{\rho-\rho_{0}}\right)_{-\mathbf{k}} \hat{V}_{\mathrm{I}}(k)\left(\widehat{\rho-\rho_{0}}\right)_{\mathbf{k}}\right. \\
& +\frac{\chi}{\gamma+2} \hat{\rho}_{-\mathbf{k}}\left(\widehat{\left.\frac{\rho}{\rho_{0}}\right)_{\mathbf{k}}^{\gamma}+1}+\left(\chi \rho_{0} \frac{\widehat{\gamma+1}}{\gamma+2}-\chi \rho\right)_{\mathbf{k}}\right] \\
& \times d \Omega_{k},
\end{aligned}
$$

where $d \Omega_{k}$ is the element of surface of the shell $|\mathbf{k}|=k$ where the hat stands for the Fourier transform defined in the same way as in the nonlocal potential after equation (4). Note that this particular choice of the spectrum is not unique and has been made so that the ground state $\rho=\rho_{0}$ contributes with no internal energy to the system. It is also worth noting that with this definition, the internal energy spectrum may take negative values.

Besides the energies, there is another quantity in quantum turbulence that presents a great interest in the dynamics of quantum vortices [36-38], which is the central line helicity per unit of volume

$$
H_{\mathrm{c}}=\frac{1}{V} \int \boldsymbol{v}(\boldsymbol{r}) \cdot \boldsymbol{\omega}(\boldsymbol{r}) d^{3} r .
$$

Note that $V H_{\mathrm{c}} / \kappa^{2}$ is the total number of helicity quanta. Formally, this quantity is ill defined for a quantum vortex as the vorticity is $\delta$ supported on the filaments and the velocity is not defined on the vortex core. However, in the GP formalism, this singularity can be removed by taking proper limits [36]. We use the definition central line helicity proposed in Ref. [36] as its numerical implementation is tedious but straightforward and well behaved for vortex tangles.

\section{EVOLUTION OF QUANTUM TURBULENT FLOWS}

This section gives a brief overview about the predictions in quantum turbulence both at large and small scales, and details of the numerical methods used to run the simulations. There is also a description of the flow visualization in the presence of a nonlocal interaction potential, and the results of the flow evolution at moderate and high resolution are shown. In particular, it is studied the dependence of the different components of the energy and the helicity with beyond mean field parameters and with the introduction of a nonlocal interaction potential.

\section{A. A brief overview of cascades in quantum turbulence}

Quantum turbulence is characterized by the disordered and chaotic motion of a superfluid. Energy injected, or initially contained, at large scales is transferred towards small scales in a Richardson cascade process [16]. In the context of GP turbulence, the contribution of vortices to the global energy can be studied by looking at the incompressible kinetic energy $E_{\text {kin }}^{I}$ and its associated spectrum. As the system evolves, vortices interact transferring energy between scales. Besides, the incompressible kinetic energy is transferred to the quantum, internal and compressible energy through vortex reconnections and sound emission [17,22]. After some time, acoustic excitations thermalize and act as a thermal bath providing a (pseudo)dissipative mechanism, so vortices shrink until they vanish $[8,39,40]$.

Three-dimensional quantum turbulence presents two main statistical properties. At scales much larger than the intervortex distance $\ell$, but much smaller than the integral scale $L_{0}$, the quantum character of vortices is not important and we can think as the system being coarse grained. At such scales the system presents a behavior that resembles classical turbulence with a direct energy cascade, that is the transfer of energy from large to small structures. As a consequence, in this range, the incompressible kinetic energy spectrum $E_{\text {kin }}^{I}(k)$ follows the Kolmogorov prediction [11,16,41,42]

$$
E_{\mathrm{kin}}^{I}(k)=C_{K} \epsilon^{2 / 3} k^{-5 / 3},
$$

where $C_{K} \sim 1$ and $\epsilon$ is the dissipation rate of the flow, which in GP quantum turbulence is associated with the rate of change of incompressible kinetic energy $\epsilon=-d E_{\text {kin }}^{I} / d t$, that is expressed in units of $[\epsilon]=$ length ${ }^{2} /$ time $^{3}$.

In classical three-dimensional inviscid flows, helicity (18) is also conserved. Associated to this invariant, a second direct cascade is expected to be also present at large scales, obeying the scaling [43]

$$
H(k)=C_{H} \eta \epsilon^{-1 / 3} k^{-5 / 3},
$$

where $C_{H} \sim 1$ and $\eta=-d H / d t$ is the dissipation rate of helicity. This dual cascade has been also observed in quantum turbulent flows described by the GP equation [44]. 
At scales smaller than the intervortex distance, each quantum vortex can be thought as if it were isolated. Hence, its behavior can be described, in principle, by the wave turbulence theory as such vortices admit hydrodynamic excitations known as Kelvin waves. Such waves propagate along vortices and interact nonlinearly among themselves. As a result, energy is transferred towards small scales through a process that can be described by the theory of weak wave turbulence [35]. An agitated debate arose some time ago concerning the prediction of the energy spectrum. Two independent groups leaded by L'vov and Nazarenko [30] and Kozik and Svistunov [45], starting from the same equations and applying the same theory derived different predictions. Even though, today there is more numerical data supporting L'vov and Nazarenko prediction [46-49], this issue is still debated [50-52]. We present here the L'vov and Nazarenko prediction as, we will see later, it was found to be in agreement with our numerical data. This theoretical prediction is derived for an almost straight vortex of period $L_{\mathrm{v}}$ and, as discussed in Ref. [50], some care is needed in order to apply the model to a turbulent vortex tangle. We partially reproduce here and adapt to our case the considerations of Ref. [50]. The wave turbulence L'vov and Nazarenko prediction is

$$
e_{\mathrm{KW}}(k)=C_{\mathrm{LN}} \frac{\kappa \Lambda \epsilon_{\mathrm{KW}}^{1 / 3}}{\Psi^{2 / 3} k^{5 / 3}},
$$

with $\Lambda=\log (\ell / \xi)$ and $C_{\mathrm{LN}} \approx 0.304$ [47]. Here $\epsilon_{\mathrm{KW}}=$ $-d e_{\mathrm{KW}} / d t$ is the mean energy flux per unit of length $L_{\mathrm{V}}$ and density $\rho_{0}$. Note their respective dimensions are $\left[\epsilon_{\mathrm{KW}}\right]=$ length ${ }^{4} /$ time $^{3}$ and $\left[e_{\mathrm{KW}}(k)\right]=$ length $^{5} /$ time $^{2}$. The dimensionless number $\Psi$ is given by

$$
\Psi=\frac{\left(12 \pi C_{\mathrm{LN}}\right)^{3 / 5} \epsilon_{\mathrm{KW}}{ }^{1 / 5}}{\kappa^{3 / 5} k_{\mathrm{min}}^{2 / 5}}=C_{\mathrm{LN}}^{3 / 5} \tilde{\Psi},
$$

where $k_{\min }$ is the smallest wave number of the Kelvin waves that can be associated with the wave number of the intervortex distance $k_{\ell}=2 \pi / \ell$ in the case of a vortex tangle [50]. $\tilde{\Psi}$ is defined so that it is independent of the constant $C_{\mathrm{LN}}$.

In order to compare this result with the incompressible kinetic energy, one can notice that the total energy of Kelvin waves is $L_{\mathrm{v}} \rho_{0} \int e_{\mathrm{KW}}(k) d k$, where now $L_{\mathrm{v}}$ is taken as the total vortex length in the system. As in a turbulent tangle the total vortex length is related to the mean intervortex distance by $L_{\mathrm{v}}=V \ell^{-2}$, it follows that the mean kinetic energy spectrum per unit of mass is given by $E_{\mathrm{KW}}(k)=e_{\mathrm{KW}}(k) \ell^{-2}$. The same logic relates the energy flux $\epsilon_{\mathrm{KW}}$ of the Kelvin wave cascade to the global energy flux $\epsilon$ of a tangle by $\epsilon_{\mathrm{KW}}=\epsilon \ell^{2}$. It follows from (21) and the previous considerations that

$$
E_{\mathrm{KW}}(k)=C_{\mathrm{LN}}^{3 / 5} \frac{\kappa \Lambda \epsilon^{1 / 3} \ell^{-4 / 3}}{\tilde{\Psi}^{2 / 3} k^{5 / 3}} .
$$

Here we have made the assumption that the energy flux in the Kolmogorov range is the same as in the Kelvin wave cascade. This strong assumption might be questioned as energy could be already dissipated into sound by vortex reconnections at different scales diminishing this value [22,53]. Such extra sinks of energy are difficult to quantify and we will not take them into account. Finally, note that the theory of wave turbulence also predicts the value of the constant $C_{\mathrm{LN}}$ [47], however in (23) several phenomenological considerations have been made and we do not expect an exact agreement. Nevertheless, the scaling with the global energy flux should remain valid.

\section{B. Numerical methods}

We perform numerical simulations of equation (7) using a pseudo-spectral method for the spatial resolution applying the " $2 / 3$ rule" for dealiasing [54], and a Runge-Kutta method of fourth order for the time stepping. The nonlinear term is dealiased twice following the scheme presented in [40] in order to also conserve momentum. Note that in the case of a nonlocal potential, this extra step has no extra numerical cost. All simulations were performed in a cubic $L$-periodic domain.

To observe a Kolmogorov range in GP turbulence it is customary to start from an initial vortex configuration with a minimal acoustic contribution. We prepared the initial condition by a minimization process such that the resulting flow is as close as possible to the targeted velocity field [11]. In this work we study the quantum Arnold-Bertrami-Childress (ABC) flow [44]. It is obtained from the velocity field $\boldsymbol{v}_{\mathrm{ABC}}=$ $\boldsymbol{v}_{\mathrm{ABC}}^{\left(k_{1}\right)}+\boldsymbol{v}_{\mathrm{ABC}}^{\left(k_{2}\right)}$, where each ABC flow is given by

$$
\begin{aligned}
\boldsymbol{v}_{\mathrm{ABC}}^{(k)}= & {[B \cos (k y)+C \sin (k z)] \hat{x}+[C \cos (k z)} \\
& +A \sin (k x)] \hat{y}+[A \cos (k x)+B \sin (k y)] \hat{z} .
\end{aligned}
$$

We set in this work $(A, B, C)=V_{\text {amp }}(0.9,1,1.1) / \sqrt{3}$, with $V_{\text {amp }}=0.5 c$. Each ABC flow is an $L$-periodic stationary solution of the Euler equation with maximal helicity, in the sense that $\nabla \times \boldsymbol{v}_{\mathrm{ABC}}^{(k)}=k \boldsymbol{v}_{\mathrm{ABC}}^{(k)}$. The mean kinetic energy associated with $\boldsymbol{v}_{\mathrm{ABC}}$ is $E_{\mathrm{kin}}^{\mathrm{ABC}}=\left(A^{2}+B^{2}+C^{2}\right)=0.2517 c^{2}$. Following Ref. [44], the wave function associated to this ABC flow is generated as $\psi_{\mathrm{ABC}}=\psi_{\mathrm{ABC}}^{\left(k_{1}\right)} \times \psi_{\mathrm{ABC}}^{\left(k_{2}\right)}$, where each mode is constructed as the product $\psi_{\mathrm{ABC}}^{(k)}=\psi_{A, k}^{x, y, z} \times \psi_{B, k}^{y, z, x} \times \psi_{C, k}^{z, x, y}$ with

$$
\psi_{A, k}^{x, y, z}=\exp \left\{i\left[\frac{A \sin (k x)}{c \xi \sqrt{2}}\right] \frac{2 \pi y}{L}+i\left[\frac{A \cos (k x)}{c \xi \sqrt{2}}\right] \frac{2 \pi z}{L}\right\}
$$

where the brackets [ ] indicate the integer closest to the value to ensure periodicity. This ansatz gives a good approximation for the phase of the initial condition. In order to set properly the mass density and the vortex profiles, it is necessary to first evolve $\psi_{\mathrm{ABC}}$ using the generalized advected real Ginzburg-Landau equation (imaginary time evolution in a locally Galilean transformed system of reference) [11]

$$
\begin{aligned}
\partial_{t} \psi= & -\frac{c_{0}}{\xi_{0} \sqrt{2}}\left\{-\xi_{0}^{2} \nabla^{2} \psi-(1+\chi) \psi+\chi \frac{|\psi|^{2(1+\gamma)}}{\rho_{0}^{1+\gamma}} \psi\right. \\
& \left.+\frac{\psi}{\rho_{0}}\left(V *|\psi|^{2}\right)\right\}-i \boldsymbol{v}_{\mathrm{ABC}} \cdot \nabla \psi-\frac{\left(\boldsymbol{v}_{\mathrm{ABC}}\right)^{2}}{2 \sqrt{2} c \xi} \psi \cdot
\end{aligned}
$$

This equation is dissipative and its final state contains a minimal amount of compressible energy. This state is used as an initial condition for the gGP equation. Unless stated otherwise, we use a flow at the largest scales of the systems by setting $k_{1}=2 \pi / L$ and $k_{2}=4 \pi / L$ throughout this work.

The numerical simulations performed in this work are summarized in Table I and regrouped in two different sets. The first set of simulations (runs A1-A8) have been performed at a moderate spatial resolution of $N^{3}=256^{3}$ grid points to study 
TABLE I. Table with the parameters of the different simulations. $N$ is the linear spatial resolution, $\chi$ and $\gamma$ are the amplitude and order of the beyond mean field interactions, $L / \xi$ is the scale separation between the domain size $L$ and the healing length $\xi, \tilde{k}_{1}=k_{1} L / 2 \pi$ and $\tilde{k}_{2}=k_{2} L / 2 \pi$ are the two wave numbers where the energy is concentrated for the initial condition, and a local or a nonlocal interaction potential is used in each of them.

\begin{tabular}{|c|c|c|c|c|c|c|}
\hline & $N$ & $\chi$ & $\gamma$ & $L / \xi$ & $\tilde{k}_{1}, \tilde{k}_{2}$ & $\begin{array}{c}\text { Interaction } \\
\text { potential }\end{array}$ \\
\hline A1 & 256 & 0 & 1 & 171 & 1,2 & local \\
\hline A2 & 256 & 1 & 1 & 171 & 1,2 & local \\
\hline A3 & 256 & 3 & 1 & 171 & 1,2 & local \\
\hline A4 & 256 & 5 & 1 & 171 & 1,2 & local \\
\hline A5 & 256 & 1 & 2.8 & 171 & 1,2 & local \\
\hline A6 & 256 & 3 & 2.8 & 171 & 1,2 & local \\
\hline A7 & 256 & 5 & 2.8 & 171 & 1,2 & local \\
\hline A8 & 256 & 0.1 & 2.8 & 171 & 1,2 & nonlocal \\
\hline B1 & 512 & 0 & 1 & 341 & 1,2 & local \\
\hline B2 & 512 & 0.1 & 2.8 & 171 & 1,2 & nonlocal \\
\hline B3 & 512 & 0.1 & 2.8 & 341 & 1,2 & nonlocal \\
\hline B4 & 512 & 0.1 & 2.8 & 341 & 2,3 & nonlocal \\
\hline B5 & 512 & 0.1 & 2.8 & 341 & 3,4 & nonlocal \\
\hline B6 & 1024 & 0.1 & 2.8 & 683 & 1,2 & nonlocal \\
\hline
\end{tabular}

the effects introduced by the beyond mean field interactions and a nonlocal potential. Each of them has a different value of $\chi$ and $\gamma$ with a local potential and were compared with a single simulation with a nonlocal interaction potential. The second set (runs B1-B6) has been performed to study the scaling of the energy spectra. In these runs, we used a spatial resolution of $512^{3}$ and $1024^{3}$ grid points, different scale separations, and initial conditions. These results were also compared with the GP model.

\section{Flow visualization}

The introduction of a nonlocal potential, as mentioned in Sec. II C, allows the system to reproduce the roton minimum in the excitation spectrum (see Fig. 1). As a consequence, the density profiles close to the quantum vortices have some fluctuations around the bulk value $\rho_{0}$ (see Fig. 2). These oscillations have been studied for the profile of a two-dimensional vortex $[25,29]$ and have been also observed in three dimensions during vortex reconnections [26]. In the case of a helical vortex tangle, the roton minimum induces a remarkable pattern of density fluctuations around a vortex line. A visualization of the initial condition $\psi_{\mathrm{ABC}}$ for run B6 is displayed in Figs. 3(a) and 3(b). The red structures are isosurfaces of low density values $\rho=0.1 \rho_{0}$ and thus represent the vortex lines. The greenish rendering displays density fluctuations of the field above the bulk value $\rho_{0}$, that are only observed in the case of a nonlocal potential. In Fig. 3(a) we recognize the large scale structures of the ABC flow accompanied by some density fluctuations around the nodal lines. Figure 3(b) displays a zoom of the tangle where such fluctuations are clearly observed. Unlike the (local) GP model, density variations around a vortex line have a very specific pattern, rolling around the nodal lines in a helicoidal manner.
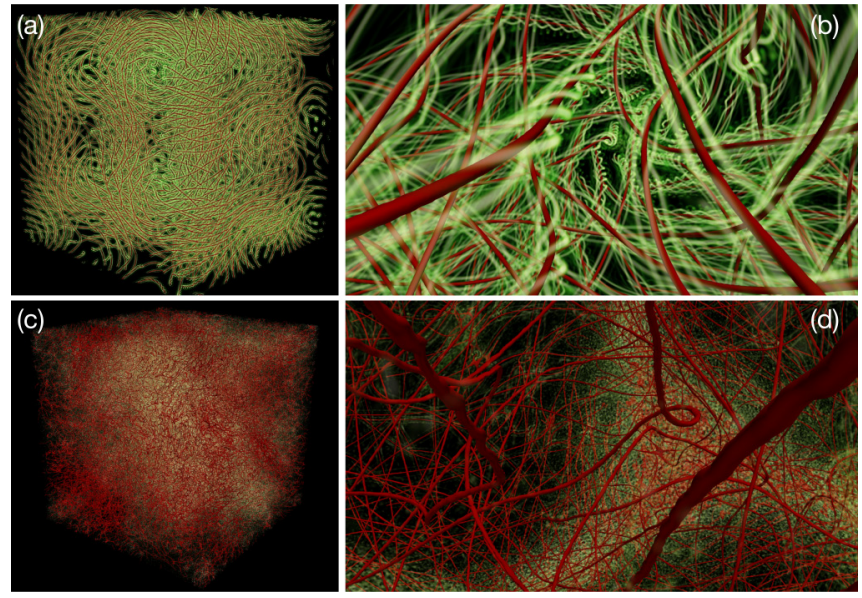

FIG. 3. (a)-(b) Visualization of an ABC flow at $t=0$ and (c)(d) for $t=1.25 \tau_{L}$ and $t=0.4 \tau_{L}$ respectively, for a resolution of $1024^{3}$ grid points with a nonlocal potential. The isosurfaces of a small value the mass density shown in red correspond to the vortex lines, and in green are the values of the density fluctuations above $\rho_{0}$.

Such a pattern is a consequence of the maximal helicity initial condition produced by the ABC flow. Indeed, we have also produced a Taylor-Green initial condition [11] that has no mean helicity, and such helicoidal patterns in the density fluctuations are absent, although they are nevertheless developed after some vortex reconnections, as observed in Ref. [26] (data not shown). Finally, in Figs. 3(c) and 3(d) we display visualizations of the field for times $t=1.25 \tau_{L}$ and $t=0.4 \tau_{L}$, respectively. Time is expressed in units of the large-eddy turnover time $\tau_{L}=L_{0} / v_{\text {rms }}$ with $v_{\text {rms }}=\sqrt{2 E_{\text {kin }}^{I}(t=0) / 3}$ and $L_{0}$ its integral length scale given by $L_{0}=2 \pi / k_{2}$ with $k_{2}$ the largest wave number used to generate the initial condition. $t=$ $1.25 \tau_{L}$ corresponds to a time when turbulence is developed and $t=0.4 \tau_{L}$ to an early stage of the turbulent development for a better insight of the flow. As the system evolves, acoustic emissions are produced and the density fluctuations increase. In Fig. 3(c) we observe a turbulent tangle where a large scale structure is predominant. Figure 3(d) displays a zoom where reconnections and Kelvin waves propagating along vortices are clearly visible.

\section{Temporal evolution of global quantities}

In this section we study the behavior of the global quantities of an $\mathrm{ABC}$ flow driven by the gGP model (7) with both local and nonlocal potentials corresponding to runs $\mathrm{A}$ in Table I. Figure 4 shows the time evolution of the (a) incompressible kinetic energy and (b) the sum of the quantum, internal, and compressible kinetic components to the total energy. We notice that in Fig. 4(a) the values of amplitude and exponent of the beyond mean field interaction and the inclusion of roton minimum (runs A1-A8) have a negligible impact on the incompressible energy of the initial condition, and their effect is very small during the temporal evolution. On the other hand, as the fluid can be considered to be more incompressible due to stronger interactions, the density variations with respect to the bulk value $\rho_{0}$ yield larger values 

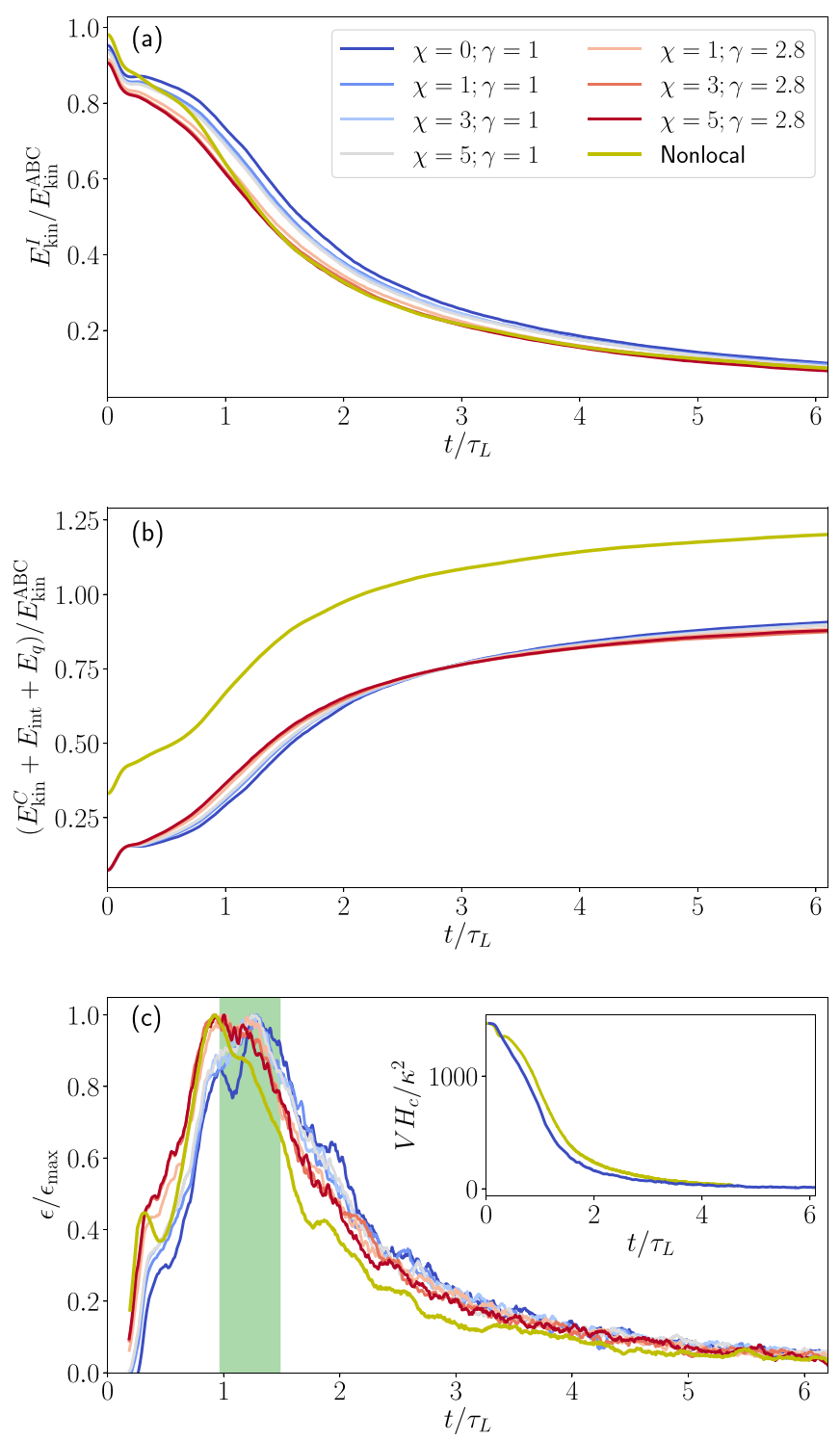

FIG. 4. Time evolution of the (a) incompressible kinetic energy, (b) the sum of the internal, quantum, and compressible kinetic energy, and (c) the dissipation rate of incompressible energy for runs $\mathrm{A}$ in Table I. The inset in (c) shows the evolution of the central line helicity. The green area corresponds to the window where the dissipation achieves a maximum and where the time averages were performed.

of the other energy component between the initial time and $t \approx 3 \tau_{L}$ as displayed in Fig. 4(b). In particular, for the case of a nonlocal potential their values remain larger for the whole run. Nevertheless, for all runs during the first large-eddy turnover times the main contribution to energy comes from vortices. At later times, energy from vortices is converted into sound. As stated in Sec. III A, the decay of the incompressible energy can be used to estimate the energy dissipation rate $\epsilon$. Its temporal evolution is displayed in Fig. 4(c). As in classical decaying turbulent flows, for quantum flows the Kolmogorov regime is more developed at times slightly after the maximum of dissipation is reached. The green zone in the figure depicts the temporal window where the system is considered to be in

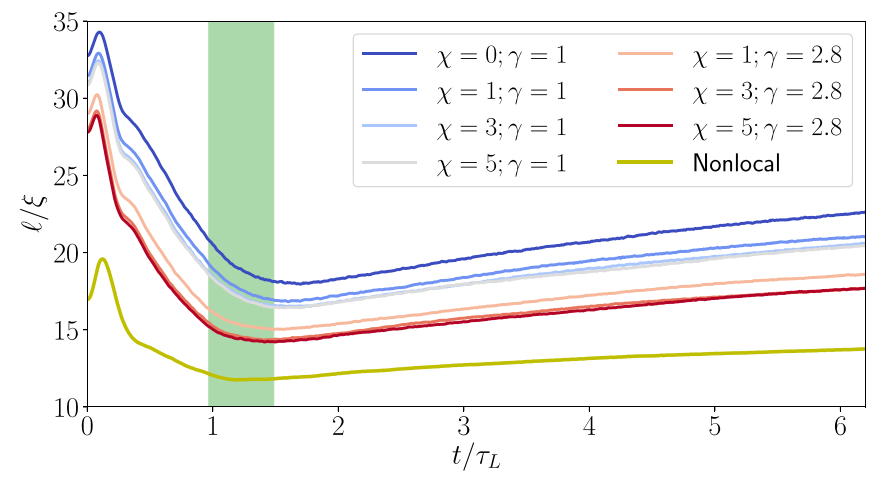

FIG. 5. Time evolution of the intervortex distance of the system in units of the healing length. All curves correspond to the runs A in Table I. The green area corresponds to the window where the dissipation achieves a maximum and where the time averages were performed.

a quasisteady state and a temporal average can be performed to improve statistics. The inset of Fig. 4(c) displays that the decay of the central line helicity is independent of the parameters of the gGP model and is consistent with the one reported in Ref. [44].

As a turbulent flow evolves, the total vortex length $L_{\mathrm{v}}$ varies in time in a competition between the vortex line stretching and the reconnection process. This quantity can be obtained from the incompressible momentum density of the flow $J^{I}(k)$ and of a two-dimensional point-vortex $J_{\text {vort }}^{2 \mathrm{D}}(k)$ as $L_{\mathrm{v}}=2 \pi \sum_{k<k_{\max }} k^{2} J^{I}(k) / \int_{0}^{k_{\max }} k^{2} J_{\mathrm{vort}}^{2 \mathrm{D}}(k) d k[11]$.

Figure 5 shows the time evolution of the intervortex distance $\ell=\sqrt{V / L_{\mathrm{v}}}$. In the cases of a local and a nonlocal interaction, the intervortex distance achieves a minimum around one $\tau_{L}$. The vortex line density of the system $\mathcal{L}=\ell^{-2}$ is expected to decay in time following either the Vinen's decay law [55] $\mathcal{L} \sim t^{-1}$, when flow is dominated by random rings, or the "quasiclassical" $\mathcal{L} \sim t^{-3 / 2}$ regime, when it is saturated by the container size [56]. In the GP framework, the Vinen's law has been clearly observed at very late times, where only a few rings are left [49]. To perform a clean study of the decay of the vortex line density, it is necessary to study the evolution of the system for much longer times that the ones presented in this work. In addition, the method implemented for the computation of the vortex length of the system is just an estimation and a more precise method needs be used to determine the scaling of the vortex length decay. Such a study is left for a future work.

Finally, in Fig. 6 we display the energy spectra for different runs of set A. Figure 6(a) shows the spectra of the incompressible kinetic energy and the sum of all the other components for different runs. Even though the range of scales is rather limited for this set of simulations, a Kolmogorov-like power law at large scales is observed in the incompressible kinetic energy. The spectra of the sum of the other energy components can be considered as the contribution of excitations that do not arise from vortices. Phenomenologically, we can consider that dynamics of the system is governed by vortices and is thus almost incompressible for scales down to the crossover between the two spectra plotted in Fig. 6(a). Such crossover wave 

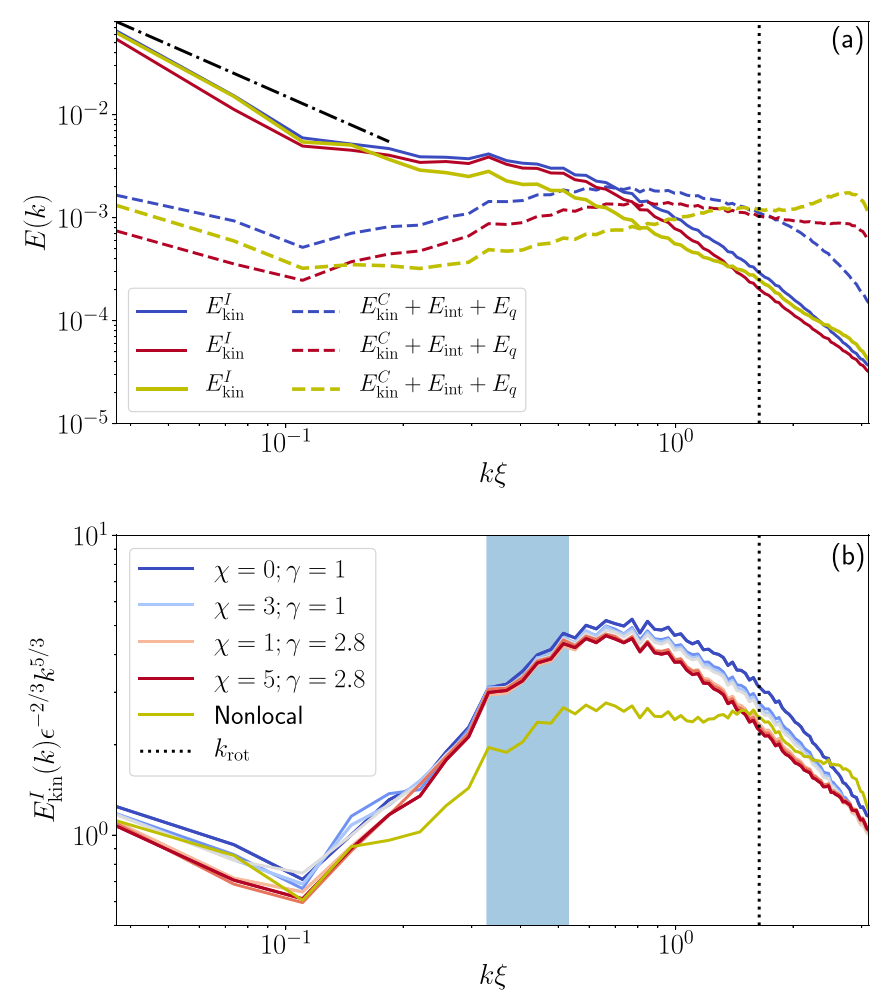

FIG. 6. (a) Time averaged spectra of the incompressible kinetic energy and the sum of the internal, quantum, and compressible kinetic energy for runs A1 (blue), A7 (red), and A8 (yellow) in Table I. (b) Compensated incompressible energy spectra for all the set of runs A. The filled blue area indicates the intervortex wave numbers $k_{\ell} \xi$ for the different simulations.

number is decreased while introducing beyond mean field terms and a nonlocal potential. Figure 6(b) displays the incompressible energy spectra compensated by the Kolmogorov prediction $E_{\text {kin }}^{I} \sim \epsilon^{2 / 3} k^{-5 / 3}$, where large scales collapse to values close to one. Remarkably, for the nonlocal potential run, a secondary plateau appears at smaller scales, below the intervortex distance (intervortex wave numbers for each run vary within the blue area). This range can be associated to the presence of Kelvin waves and it will be studied at higher resolutions in the next section.

\section{E. Numerical evidence of the coexistence of Kolmogorov and Kelvin wave cascades}

The Kelvin wave cascade discussed in Sec. III A is formally derived from an incompressible model in a very simplified theoretical setting. In the context of the GP model, the Kelvin wave cascade was first observed in Ref. [46] where a setting close to the theoretical prediction was used. In the case of turbulent tangles, there was first an indirect observation of the Kelvin wave cascade by making use of the spatiotemporal spectra [57]. In that work, the Kelvin wave dispersion relation was glimpsed and a space-time filtering of the fields was performed yielding a scaling in the energy spectrum compatible to the Kelvin wave cascade. Then, by using an accurate tracking algorithm of a turbulent tangle, in Ref. [49] the L'vov-Nazarenko prediction was clearly observed in the
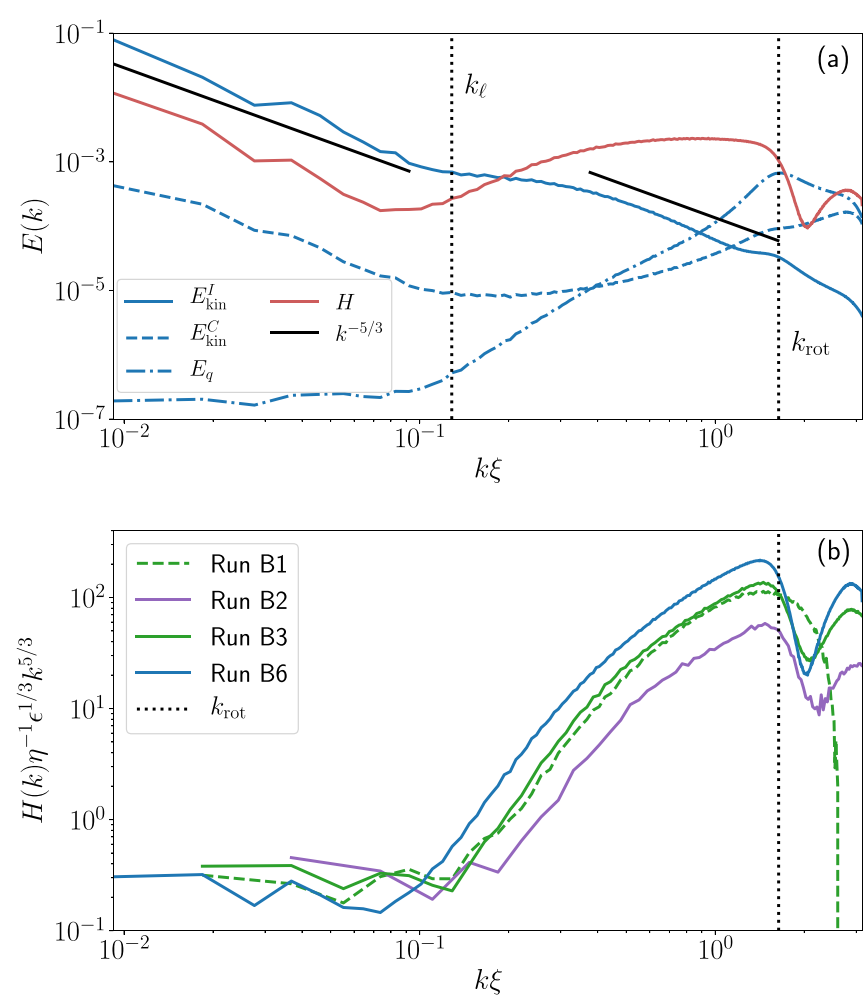

FIG. 7. (a) Helicity and energy spectra of the different components for the simulation with $1024^{3}$ grid points (run B6). Vertical dotted lines indicate the wave numbers associated with the intervortex distance $k_{\ell}$ and the roton minimum $k_{\text {rot }}$. (b) Helicity spectra compensated by Eq. (20) for runs B1-B3 and B6 shown in Table I.

spectrum of large vortex rings extracted from the tangle. Later, in Refs. [13,44], by using high-resolution numerical simulations of the GP model, and by superposing different runs, a secondary scaling range compatible with Kelvin wave cascade predictions was observed. In this section, we focus on the scaling of the incompressible energy spectra and helicity for the case with a nonlocal potential (set of runs B) as it seems to present a much clearer scaling at scales smaller than the intervortex distance. We vary different parameters so the range of scales (system size, intervortex distance, healing length) and energy fluxes take different values.

The spectra for the different components constituting the kinetic energy and the helicity of the simulation with $1024^{3}$ grid points are shown in Fig. 7(a). Clear power laws for the Kolmogorov and Kelvin wave range are observed simultaneously at large and small scales. A fit $k^{-\alpha}$ using the least squares method was performed for each cascade. We obtain a scaling $\alpha=1.73$ in the range between $k \xi=0.02$ and $k \xi=$ 0.12 , associated with the Kolmogorov cascade, and a scaling $\alpha=1.65$ in the range between $k \xi=0.33$ and $k \xi=1.64$, associated with a Kelvin wave cascade. These two scaling laws are separated by the intervortex wave number $k_{\ell}$. At this scale, a bottleneck between a strong and a weak cascade takes place and a plateau in the incompressible kinetic energy is observed. According to the warm cascade ideas [58], this bottleneck should display a $k^{2}$ scaling associated with the thermalization of the system. However, this behavior is not observed probably due to the fact that the separation of scales 
TABLE II. Values of integral scale $L_{0}$, the quantum of circulation $\kappa$, the energy dissipation rate $\epsilon$, and the intervortex distance, expressed in units of the box size $L=2 \pi$, and the speed of sound is fixed to $c=1$.

\begin{tabular}{cccccc}
\hline \hline Run & $v_{\text {rms }}$ & $L_{0}$ & $\kappa$ & $\epsilon$ & $\ell$ \\
\hline B1 & 0.395 & $L / 2$ & 0.163 & 0.012 & 0.412 \\
B2 & 0.377 & $L / 2$ & 0.327 & 0.013 & 0.494 \\
B3 & 0.398 & $L / 2$ & 0.163 & 0.012 & 0.255 \\
B4 & 0.406 & $L / 3$ & 0.163 & 0.020 & 0.235 \\
B5 & 0.403 & $L / 4$ & 0.163 & 0.029 & 0.227 \\
B6 & 0.392 & $L / 2$ & 0.081 & 0.011 & 0.139 \\
\hline \hline
\end{tabular}

in the Kelvin wave range is not large enough. We will come back to this point later.

Concerning other energy components, the quantum energy shows a maximum at the scale associated with the roton minimum, whereas its contribution is negligible at large scales. The helicity spectrum also displays a Kolmogorov-like behavior at large scales, while at scales between the intervortex distance and the roton minimum it flattens. This flat range of the helicity spectrum appears in the range where the Kelvin wave cascade is dominant. Whether a direct relationship between the Kelvin wave cascade and the flattening of the central line helicity spectrum exists is still unclear. Figure 7(b) displays the compensated helicity spectrum according to (20) for different runs displaying different scale separations and with local and nonlocal potentials. The parameters of these simulations correspond to the runs B1-B3 and B6 shown in Table I. At large scales all curves collapse to a constant $C_{H} \sim 1$, while at smaller scales the system with a wider scale separation displays that the helicity contribution is more intense.

To analyze further the incompressible energy spectra, we have performed two runs varying the integral length of the initial condition so that the dissipation rate also changes (runs B4 and B5). We recall that in classical turbulence, the energy flux $\epsilon$ is fixed by the inertial range and varies as $\epsilon \sim v_{\mathrm{rms}}^{3} / L_{0}$. Our initial condition $\psi_{\mathrm{ABC}}$ keeps fixed, by construction, the value of $v_{\text {rms. }}$. In Table II we present the values of different physical quantities relevant for a turbulent state. Such quantities are expressed, as customary in classical turbulence, in units of large scale quantities. In particular, the system size is $L=2 \pi$ and the speed of sound is $c=1$. With such definitions, large scale quantities remain almost constant when increasing the scale separation between the box size and the smallest scale of the system, but the quantum of circulation takes smaller values.

Figure 8(a) shows the incompressible energy spectra compensated by $k^{-5 / 3}$. Two plateaux are clearly observed: one corresponding to the large scales Kolmogorov scaling and the other small-scale Kelvin wave cascade. It can be seen that the values of these plateaux differ by a factor of 3 . By following the procedures introduced in Ref. [58], but using prediction of Eq. (23) for the Kelvin wave spectrum, the ratio between these two plateaux is expected to scale as

$$
E_{K W} / E_{K 41} \sim \Lambda^{22 / 15},
$$

where we recall that $\Lambda=\log \ell / \xi$. The previous derivation assumes that $\Lambda \gg 1$. In our simulations, this quantity takes a
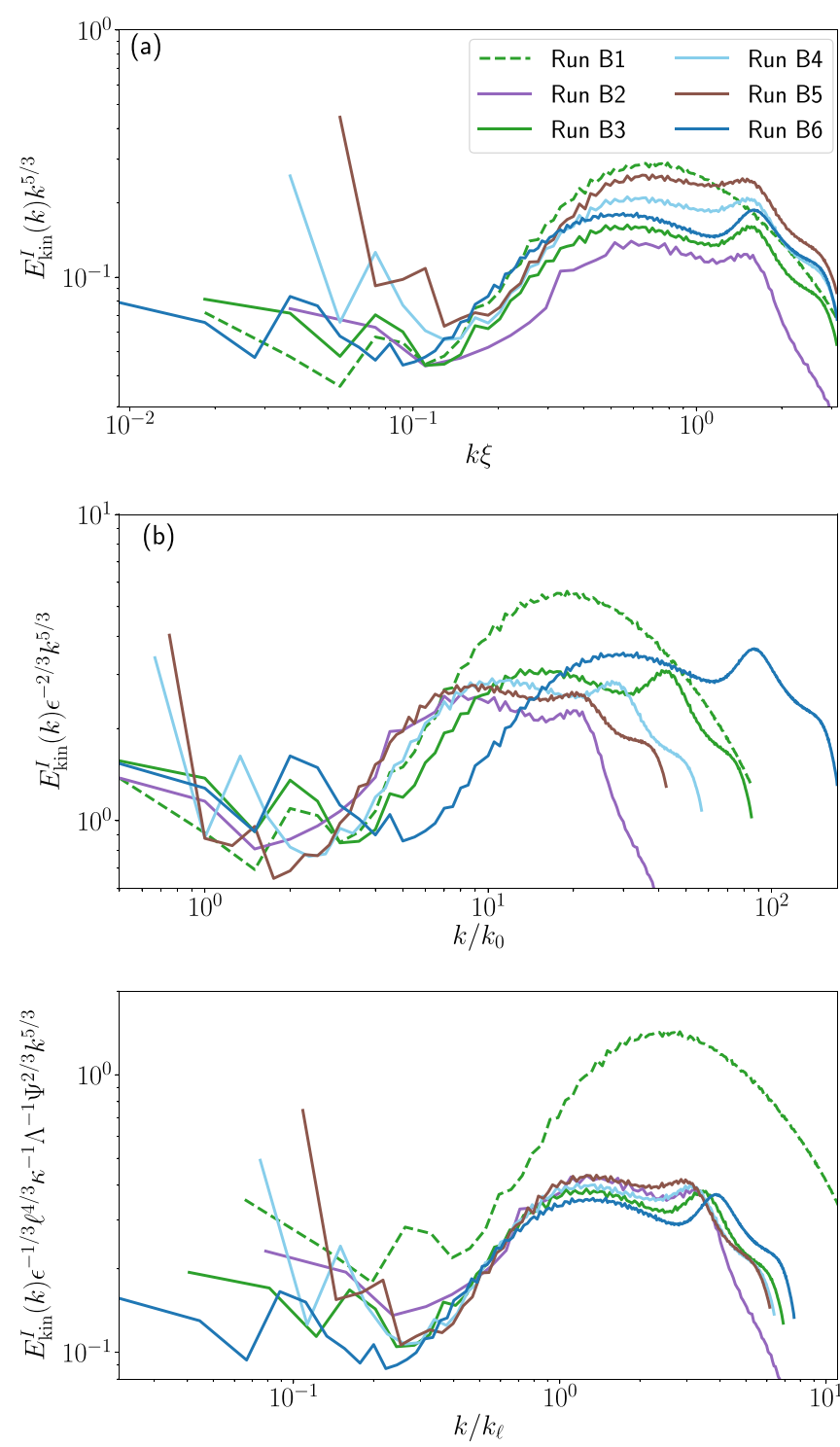

FIG. 8. Compensated incompressible kinetic energy spectra by (a) $k^{-5 / 3}$ scaling, (b) Kolmogorov scaling (19). and (c) L'vovNazarenko scaling for Kelvin waves (23).

value of $\Lambda \approx 2.7$, which cannot be considered large to apply the previous formula safely. It is nevertheless expected that if the inertial range of the Kelvin wave cascade is extended, the bottleneck joining the two regimes should be enhanced, eventually leading to a thermalized $k^{2}$ zone.

The energy spectra shown in Fig. 8(b) have been compensated by the Kolmogorov law (19) and displayed as a function of $k / k_{0}$, with $k_{0}=2 \pi / L_{0}$ in order to emphasize the Kolmogorov regime. Once properly normalized, all runs present a plateau at large scales that collapse to values that fluctuate around a Kolmogorov constant $C_{K} \sim 1$, in agreement with previous simulations of the GP model [13,44]. In order to emphasize the Kelvin wave cascade, we make use of the L'vov and Nazarenko wave turbulence prediction (23). Figure 8(c) displays the incompressible energy spectra compensated by this theoretical prediction as a function of $k / k_{\ell}$, with $k_{\ell}=2 \pi / \ell$. The collapse of the Kelvin wave 
cascade is remarkable. All runs having a nonlocal potential display a plateau around a value $C_{\mathrm{LN}}^{3 / 5} \approx 0.36$, which recovers a constant of $C_{\mathrm{LN}} \approx 0.18$. Such a value is relatively close to the predicted one $C_{\mathrm{LN}}=0.304$, in particular considering all the phenomenological assumptions made in Sec. III A to adapt the theoretical prediction (21) to the case of a turbulent tangle in Eq. (23). It is also important to remark that Eq. (21) is obtained from the Biot-Savart model, while the dynamics studied in this work corresponds to the gGP model, with a nonlocal interaction potential and beyond mean field corrections. The GP run (with local interaction potential) displays a good Kolmogorov scaling at large scales. However, it does not clearly exhibit a Kelvin wave cascade range at the highest resolution used in this work for this model $\left(512^{3}\right.$ grid points). Note that previous works reporting a secondary $k^{-5 / 3}$ range in local GP model after superposing different runs have used resolutions of $2048^{3}$ and $4096^{3}$ collocation points [13,44]. For the sake of completeness, the incompressible kinetic energy spectrum compensated by the Kozik and Svistunov prediction [45] is displayed in Appendix.

\section{CONCLUSIONS}

We studied the properties of the freely decaying quantum turbulence of the generalized Gross-Pitaevskii (gGP) model (7) that includes beyond mean field corrections and considers a nonlocal interaction potential between bosons. This model pretends to give a better description of superfluid helium as it reproduces a roton minimum in the excitation spectrum.

The visualization of the flow with a nonlocal potential allowed us to observe the formation of helical structures around the vortices produced by density fluctuations, exhibiting the intrinsic property of maximal helicity of an $\mathrm{ABC}$ flow. These structures were not observed at initial times in a flow with no helicity like a Taylor-Green flow, but they develop as the system evolves (data not shown). However, it was seen that the behavior of the helicity is independent of the interaction potential. At large scales the helicity develops a spectrum that satisfies prediction (20), while at scales between the intervortex distance and the healing length a plateau is observed. This range is usually associated with the Kelvin wave cascade regime, but it is still not clear whether the formation of this plateau is associated with Kelvin waves or not.

By studying numerically the freely decaying quantum turbulence of an $\mathrm{ABC}$ flow, we observed that the statistical behavior of the system does not depend much on the parameters of the beyond mean field correction in the presence of a local interaction potential between bosons. This is manifest in the evolution of quantities such as the energy, the helicity, and the intervortex distance of the system. The introduction of a nonlocal potential does not modify significantly the behavior of the system at large scales, exhibiting a Kolmogorov-like scaling law for the incompressible kinetic energy. However, the situation changes at smaller scales when a nonlocal potential is implemented, between the intervortex distance $\ell$ and the healing length $\xi$, the range associated with the Kelvin wave cascade. Here, the nonlocal potential enhances a second scaling of the incompressible energy spectrum. This is observed even at a moderate resolution of $256^{3}$ grid points, while in the case of a local GP model an energy spectrum compatible with $k^{-5 / 3}$ scaling law begins to be recognizable from resolutions of $2048^{3}$ collocation points [44], and even in this case the range of scales where it takes place is less than a decade. This stronger manifestation of the Kelvin wave cascade may be very useful for numerical and theoretical studies of wave turbulence. This clear difference with the local GP model may be used to compare if effectively this model better describes the dynamics of superfluid helium. However, experimental observation at scales smaller than the intervortex distance still remains a challenge.

We also studied the scaling of the Kelvin wave spectrum with the energy flux $\epsilon$ and the intervortex distance by varying the integral scale of the initial flow and its healing length. We observed that the different spectra tend to collapse to a constant according to L'vov and Nazarenko spectrum for Kelvin waves (23). The observed value of the constant is $C_{\mathrm{LN}} \approx 0.18$ which is close to the predicted one $C_{\mathrm{LN}} \approx 0.304$. This is surprising given that the theory is constructed using a Biot-Savart model considering a single vortex line, while here it is extended to a vortex tangle in the framework of the gGP model with a nonlocal interaction potential and including several phenomenological assumptions. The Kozik and Svistunov spectrum for Kelvin waves was also studied for these set of simulations, however, by compensating the energy spectra by this theory no clear plateau is observed (see Appendix). Furthermore, in the range of the Kelvin wave cascade the Kozik-Svistunov cascade would take values of $C_{\mathrm{KS}} \approx 0.06$ which is not of order one, so it might imply that the energy spectrum is not described by this theory.

The overall results of this work show that both GP and gGP models describe a similar behavior at large scales, exhibiting a classical Kolmogorov law for the incompressible kinetic energy and helicity spectra. However, at small scales, the gGP model includes the roton minimum in the excitation spectrum, and the Kelvin wave cascade range is enhanced, showing an apparent discrepancy with the local GP model. In summary, the simulations of this generalized model allow for a simultaneous observation of the Kolmogorov and Kelvin wave cascades, at resolutions at which the (standard) GP model barely exhibit a Kelvin wave range. Further studies are needed to understand the effect of nonlocal interactions deeply.

\section{ACKNOWLEDGMENTS}

The authors thank U. Giuriato and J.I. Polanco for fruitful discussions. N.P.M. and G.K. were supported by the Agence Nationale de la Recherche through the project GIANTE ANR18-CE30-0020-01. Computations were carried out on the Mésocentre SIGAMM hosted at the Observatoire de la Côte d'Azur and the French HPC Cluster OCCIGEN through the GENCI allocation A0042A10385. G.K. is also supported by the EU Horizon 2020 research and innovation programme under the Grant Agreement No. 823937 in the framework of Marie Sklodowska-Curie HALT project and the Simons Foundation Collaboration grant Wave Turbulence (Award ID 651471).

\section{APPENDIX: KOZIK-SVISTUNOV KELVIN SPECTRUM}

The original Kozik and Svistunov prediction for the Kelvin wave cascade [45] was done with the same geometrical 


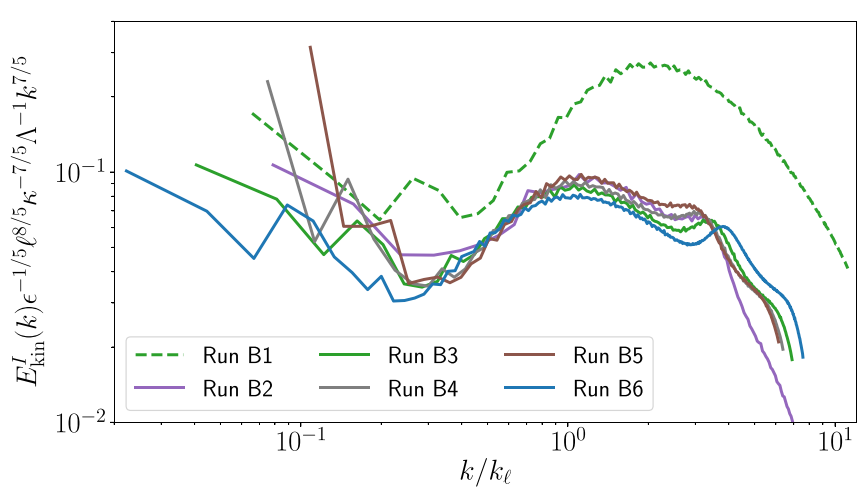

FIG. 9. Compensated incompressible kinetic energy spectra by the Kozik and Svistunov prediction for Kelvin waves.

considerations of L'vov and Nazarenko and it is also expressed in units of length ${ }^{5} /$ time $^{2}$. Applying the same considerations of Sec. III A to adapt this prediction to a turbulent three- dimensional flow leads to the following Kelvin wave energy spectrum

$$
E_{\mathrm{KW}}^{\mathrm{KS}}(k)=C_{\mathrm{KS}} \frac{\kappa^{7 / 5} \Lambda \epsilon^{1 / 5} \ell^{-8 / 5}}{k^{7 / 5}},
$$

where the constant $C_{\mathrm{Ks}}$ could be in principle determined by the theory if some integrals in the associated kinetic equation are convergent, but its value is still unknown. Figure 9 displays the incompressible kinetic energy spectrum compensated by prediction (A1). All the curves tend to collapse in the range associated with Kelvin waves, showing a proper scaling with the energy flux $\epsilon$, the intervortex distance $\ell$, and the quantum of circulation $\kappa$. However, although the Kelvin wave range is limited, a plateau is not clearly observed if the spectra are compensated by (A1), and even though a constant cannot be well defined, the energy spectra collapse to a mean value of $C_{\mathrm{KS}} \approx 0.06$, which is not of order one.
[1] L. P. Pitaevskii and S. Stringari, Bose-Einstein Condensation and Superfluidity, Vol. 164 (Oxford University Press, New York, 2016).

[2] M. H. Anderson, J. R. Ensher, M. R. Matthews, C. E. Wieman, and E. A. Cornell, Sci., New Ser. 269, 198 (1995).

[3] P. Kapitza, Nature (London) 141, 74 (1938).

[4] J. F. Allen and A. Misener, Nature (London) 141, 75 (1938).

[5] F. London, Nature (London) 141, 643 (1938).

[6] E. Fonda, D. P. Meichle, N. T. Ouellette, S. Hormoz, and D. P. Lathrop, Proc. Natl. Acad. Sci. 111, 4707 (2014).

[7] S. Serafini, M. Barbiero, M. Debortoli, S. Donadello, F. Larcher, F. Dalfovo, G. Lamporesi, and G. Ferrari, Phys. Rev. Lett. 115, 170402 (2015).

[8] C. F. Barenghi, L. Skrbek, and K. R. Sreenivasan, Proc. Natl. Acad. Sci. 111, 4647 (2014).

[9] K. W. Schwarz, Phys. Rev. B 38, 2398 (1988).

[10] J. Koplik and H. Levine, Phys. Rev. Lett. 71, 1375 (1993).

[11] C. Nore, M. Abid, and M. E. Brachet, Phys. Fluids 9, 2644 (1997).

[12] A. W. Baggaley, J. Laurie, and C. F. Barenghi, Phys. Rev. Lett. 109, 205304 (2012).

[13] V. Shukla, P. D. Mininni, G. Krstulovic, P. C. di Leoni, and M. E. Brachet, Phys. Rev. A 99, 043605 (2019).

[14] J. Maurer and P. Tabeling, Europhys. Lett. 43, 29 (1998).

[15] J. Salort, B. Chabaud, E. Lévêque, and P.-E. Roche, Europhys. Lett. 97, 34006 (2012).

[16] U. Frisch, Turbulence: The Legacy of A.N. Kolmogorov, 1st ed. (Cambridge University Press, Cambridge, 1995).

[17] W. F. Vinen, Phys. Rev. B 64, 134520 (2001).

[18] B. Rousset, P. Bonnay, P. Diribarne, A. Girard, J. M. Poncet, E. Herbert, J. Salort, C. Baudet, B. Castaing, L. Chevillard, F. Daviaud, B. Dubrulle, Y. Gagne, M. Gibert, B. Hébral, T. Lehner, P.-E. Roche, B. Saint-Michel, and M. Bon Mardion, Rev. Sci. Instrum. 85, 103908 (2014).

[19] R. J. Donnelly, Quantized Vortices in Helium II (Cambridge University Press, Cambridge, 1991).

[20] A. Villois, D. Proment, and G. Krstulovic, Phys. Rev. Fluids 2, 044701 (2017).
[21] G. Krstulovic, M. Brachet, and E. Tirapegui, Phys. Rev. E 78, 026601 (2008).

[22] A. Villois, D. Proment, and G. Krstulovic, Phys. Rev. Lett. 125, 164501 (2020).

[23] R. J. Donnelly and C. F. Barenghi, J. Phys. Chem. Ref. Data 27, 1217 (1998).

[24] Y. Pomeau and S. Rica, Phys. Rev. Lett. 71, 247 (1993).

[25] N. G. Berloff and P. H. Roberts, J. Phys. A: Math. Gen. 32, 5611 (1999).

[26] J. Reneuve, J. Salort, and L. Chevillard, Phys. Rev. Fluids 3, 114602 (2018).

[27] T. D. Lee, K. Huang, and C. N. Yang, Phys. Rev. 106, 1135 (1957).

[28] N. G. Berloff, M. Brachet, and N. P. Proukakis, Proc. Natl. Acad. Sci. 111, 4675 (2014).

[29] S. Villerot, B. Castaing, and L. Chevillard, J. Low Temp. Phys. 169, 1 (2012).

[30] V. S. L'vov and S. Nazarenko, JETP Lett. 91, 428 (2010).

[31] T. Lahaye, C. Menotti, L. Santos, M. Lewenstein, and T. Pfau, Rep. Prog. Phys. 72, 126401 (2009).

[32] A. Griesmaier, J. Phys. B: At., Mol. Opt. Phys. 40, R91 (2007).

[33] L. Santos, G. V. Shlyapnikov, and M. Lewenstein, Phys. Rev. Lett. 90, 250403 (2003).

[34] S. M. Roccuzzo, A. Gallemi, A. Recati, and S. Stringari, Phys. Rev. Lett. 124, 045702 (2020).

[35] S. Nazarenko, Wave Turbulence, Lecture Notes in Physics No. 825 (Springer, Heidelberg, 2011).

[36] P. Clark di Leoni, P. D. Mininni, and M. E. Brachet, Phys. Rev. A 94, 043605 (2016).

[37] S. Zuccher and R. L. Ricca, Phys. Rev. E 92, 061001(R) (2015).

[38] M. W. Scheeler, D. Kleckner, D. Proment, G. L. Kindlmann, and W. T. M. Irvine, Proc. Natl. Acad. Sci. 111, 15350 (2014).

[39] G. Krstulovic and M. Brachet, Phys. Rev. B 83, 132506 (2011).

[40] G. Krstulovic and M. Brachet, Phys. Rev. E 83, 066311 (2011).

[41] S. K. Nemirovskii, Phys. Rep. 524, 85 (2013).

[42] L. Skrbek and K. R. Sreenivasan, Phys. Fluids 24, 011301 (2012).

[43] A. Brissaud, Phys. Fluids 16, 1366 (1973). 
[44] P. Clark di Leoni, P. D. Mininni, and M. E. Brachet, Phys. Rev. A 95, 053636 (2017).

[45] E. V. Kozik and B. V. Svistunov, J. Low Temp. Phys. 156, 215 (2009).

[46] G. Krstulovic, Phys. Rev. E 86, 055301(R) (2012).

[47] L. Boué, R. Dasgupta, J. Laurie, V. L'vov, S. Nazarenko, and I. Procaccia, Phys. Rev. B 84, 064516 (2011).

[48] A. W. Baggaley and J. Laurie, Phys. Rev. B 89, 014504 (2014).

[49] A. Villois, D. Proment, and G. Krstulovic, Phys. Rev. E 93, 061103(R) (2016).

[50] V. Eltsov and V. S. L'vov, JETP Lett. 111, 389 (2020).

[51] E. B. Sonin, Pis'ma Zh. Eksp. Teor. Fiz. 111, 706 (2020) [JETP Lett. 111, 598 (2020)].
[52] V. B. Eltsov and V. S. L'vov, JETP Lett. 111, 600 (2020).

[53] D. Proment and G. Krstulovic, Phys. Rev. Fluids 5, 104701 (2020).

[54] D. Gottlieb and S. A. Orszag, Numerical Analysis of Spectral Methods: Theory and Applications (SIAM, Philadelphia, 1977).

[55] W. F. Vinen, Proc. R. Soc. London, Ser. A 242, 493 (1957).

[56] P. M. Walmsley and A. I. Golov, Phys. Rev. Lett. 118, 134501 (2017).

[57] P. Clark di Leoni, P. D. Mininni, and M. E. Brachet, Phys. Rev. A 92, 063632 (2015).

[58] V. S. L'vov, S. V. Nazarenko, and O. Rudenko, Phys. Rev. B 76, 024520 (2007). 